\title{
It was twenty years ago today: a celebration of two decades of optical sectioning
}

\author{
Stephen W. Paddock
}

BioTechniques 35:1156-1162 (December 2003)

\section{INTRODUCTION: "THE BLUE OF PLUMS"}

In addition to the 20-year anniversary of BioTechniques (1), 2003 has been quite a year for celebrating technical achievements. These include 50 years since the description of the DNA double helix (2), 50 years since the conquest of Mount Everest, and 100 years since the first controlled powered airplane flight. However, the tercentenary of the death of Robert Hooke is arguably the most notable milestone for light microscopy techniques.

Hooke, who died in London in 1703, is widely acknowledged to be the first person to use the word "cell" in order to describe sections of cork. Using various experimental and optical methods, including his compound microscope, he observed and recorded many different biological structures from plants, insects, sponges, bird feathers, and fossils. He documented his observations with high-resolution hand drawings and published them in his classic book Micrographia [Hooke, R. (1665) Micrographia. Octavo digital rare books. http://www.octavo.com]. The book is remarkable for its time and was generally well received. Hooke did have his critics, and he was described by one of them as someone who "has spent $£ 2000$ on microscopes to find out the nature of Eels in Vinegar, Mites in Cheese, and the Blue of Plums, which he has subtly found out to be living creatures" [Waggoner, B. (2001) Robert Hooke (1635-1703) http: //www.ucmp.berkeley.edu/history/hooke.html and Reference 3]. Some 300 years later, biologists have a staggering array of techniques available to them for probing the structure of many different organisms (4).

\section{THE RENAISSANCE OF LIGHT MICROSCOPY}

The renaissance of the light microscope really refers to the incorporation of new technology into the basic instrument over the past 20 years. The word "renaissance" is an appropriate description, because on the one hand, it gives the impression of a rebirth or revival, but it also forges a connection to art. The Renaissance period was described as one of revived intellectual, artistic achievement, and enthusiasm. The images produced by today's light microscopes not only have scientific meaning, but they can also illuminate the breathtakingly beautiful forms of cells. They often adorn the covers of books and journals (including BioTechniques) and serve to draw attention to the research, often eliciting enthusiastic responses to it (Figure 1). This is not to take anything away from the images of the past, and at least one cell biological journal continues to feature them for its cover art (3).

The major components of the light microscope have remained unchanged over the years. Indeed, for some applications, older lenses have superior optics and are preferred to their modern counterparts. The more recent contributions to the renaissance include digital imaging systems, improved detectors (video cameras, charged-coupled devices, photomultipliers), filters (acousto-optic devices), light sources (lasers), and improved probes of biological activity [green fluorescent protein (GFP), quantum dots]. These improvements and their applications have been recently reviewed $(5,6)$.

\section{THE COMING OF AGE OF CONFOCAL MICROSCOPY}

The emergence and subsequent evolution of confocal microscopy into a standard technique has epitomized the renaissance of light microscopy over the past 20 years. Marvin Minsky invented the confocal microscope almost 50 years ago specifically for studying neural networks in the living brain (7). All modern confocal microscopes are based on his original idea, which was patented in 1957 (8). However, the technology for the practical application of the confocal approach was not available to him at this time, and was only realized as a technique for biomedical research some 30 years later.

In Minsky's original design, the light source was a zirconium bulb, and scanning of the specimen was achieved by moving the stage. The image was built up on the screen of a military surplus oscilloscope. Images produced using the early microscope failed to attract attention, not because of the resolution achieved by the instrument, but because of the inferior quality of the output device.

Modern confocal microscopes are loaded with technological advancements. The light source is usually a laser, the scan- 
ning is achieved electronically under computer control, the filters are electronic, the detectors are sensitive photomultiplier tubes (PMTs) or charged-coupled devices, and the final image is either built up in a computer framestore, computer memory, or by a sensitive digital camera, and then displayed on a highresolution video monitor and recorded digitally. This results in virtually no loss of resolution along the imaging chain from the microscope to the final publication, especially as most journals are now available in a digital form on the web.

Minsky's original design was a single-beam stage-scanning instrument. This design is now used for imaging larger specimens that do not move (e.g., microarrays or whole animal sections) (9-11). An alternative to moving the specimen is to scan the beam across the specimen. This is the preferred method of scanning most biological specimens (especially living ones), because there is less chance of vibrations resulting in image deterioration. Micromanipulation is a practical option when the specimen is stationary.

There are two fundamentally different methods of beam scanning: multiple-beam scanning or single-beam scanning. The pioneer of multiple-beam technology was Mohimir Petran, who, like Marvin Minsky, was interested in imaging living growing nerve cells in the intact brain. His ingenious design, the tandem scanning reflected light microscope (TSM), incorporates a bright white light source and a modified Nipkow disc to scan the specimen with hundreds of points of light (12). The disk spins rapidly and acts both as the point source and the spatial filter that prevents out-of-focus light from reaching the photodetector. The disk is designed with apertures that are matched on the excitation and emission side of the light path. The photodetector in this case can be the human eye or a sensitive video camera mounted on the microscope. Nipkow disc scanning microscopes that have evolved from the TSM over the years have greatly improved sensitivity for fluorescence. These instruments are now available commercially and have recently become popular for viewing cytoskeletal dynamics by fluorescent speckle microscopy $(13,14)$.

The first practical single-beam scanning instrument for epifluorescence microscopy, the laser scanning confocal microscope (LSCM), was designed by John White and Brad Amos in Cambridge, England with the specific aim of imaging changes in cytoarchitecture of blastomeres during early embryogenesis of fluorescently labeled nematode embryos. The first two cycles of division were visualized relatively easily using conventional epifluorescence microscopy. However, images of later stages became uninterpretable because of glare from out-of-focus structures. When he investigated the confocal microscopes available to him at the time, White found that his options were limited. The technology consisted of the stage scanning instruments, which tended to be slow to produce images (approximately $10 \mathrm{~s}$ for one full frame image), and the multiple-beam microscopes, which were difficult to align and the fluorescence images were extremely dim.

White and his colleagues, by incorporating available technology (lasers, computer imaging systems, and related electronics) designed a LSCM for epifluorescence microscopy. This new LSCM along with images of various fluorescently labeled embryos captured with it was described in 1987 (15), and the stunning images "enraptured the world's biological community with their exquisite and convincing illustrations of the power of the confo- 
cal laser scanning microscope" (16). The LSCM has evolved into an instrument that has been applied to many different biomedical problems. The descendants of this microscope, together with several other LSCMs, are now available commercially.

The LSCM quickly found additional biomedical applications to critical confocal imaging. Many of these applications were made possible by the ability to control the scanning mirrors using the microcomputer. For example, the magnification could be changed by optically zooming using the same objective lens and with no loss in image resolution. This was achieved by decreasing the area scanned by the beam and thus allowing for a wide range of magnifications to a single objective lens. Furthermore, the scanning mirrors could be controlled to scan a line, a spot, or a shape onto the specimen for fine control of photobleaching or photoactivation, and more recently, for fluorescence resonance energy transfer (FRET), fluorescence lifetime imaging (FLIM), or fluorescence localization after photobleaching (FLAP) experiments (17-19).

Serial optical sections (Z-series) could be collected by driving the fine focus of the microscope with a stepper motor and correlating image acquisition with the movement of the stage. Since there was no physical sectioning involved, and assuming that the specimen did not move during acquisition of the images, all of the sections were in perfect register. Since the Z-series files were in a digital form, it was relatively simple to export them to a 3-D reconstruction program for further analysis and display.

The LSCM has rapidly gained popularity for multiple label imaging, since the registration between the images collected at different excitation wavelengths is maintained. Two-color imaging using fluorescein and rhodamine probes was quickly achieved, along with the ability to merge a nonconfocal transmitted light image with the fluorescence images. Threechannel imaging is now relatively routine, using a mixed gas laser (or combinations of lasers) and a scanning head with three PMTs that are able to collect the three channels simultaneously $(20,21)$. Over the past few years, there have been improvements made for imaging more than three probes in the same sample, and recent developments now allow the detection of 32 separate channels. This gives a finer control of bleed through between channels, improved rejection of autofluorescence, and the ability to separate probes whose emission spectra overlap (22)

The current fashion is to call these kinds of data, Z-series, multichannel, and time-lapse data, "multidimensional." The number of dimensions has now reached five. These dimensions are the $\mathrm{X}$, the $\mathrm{Y}$, and the $\mathrm{Z}$ planes, together with time as the fourth dimension, and wavelength as the fifth dimension. Thus, timelapse series of Z-series of multichannel images are collected with the result that huge data sets are rapidly acquired $(23,24)$.

\section{LIFE AND DEPTH: MULTIPLE-PHOTON IMAGING}

The early LSCMs were excellent at what they were designed for; namely, imaging fixed and fluorescently labeled specimens. The instruments had several drawbacks for researchers attempting to image deep into living samples. The early LSCMs were very wasteful of photons. It took a lot of
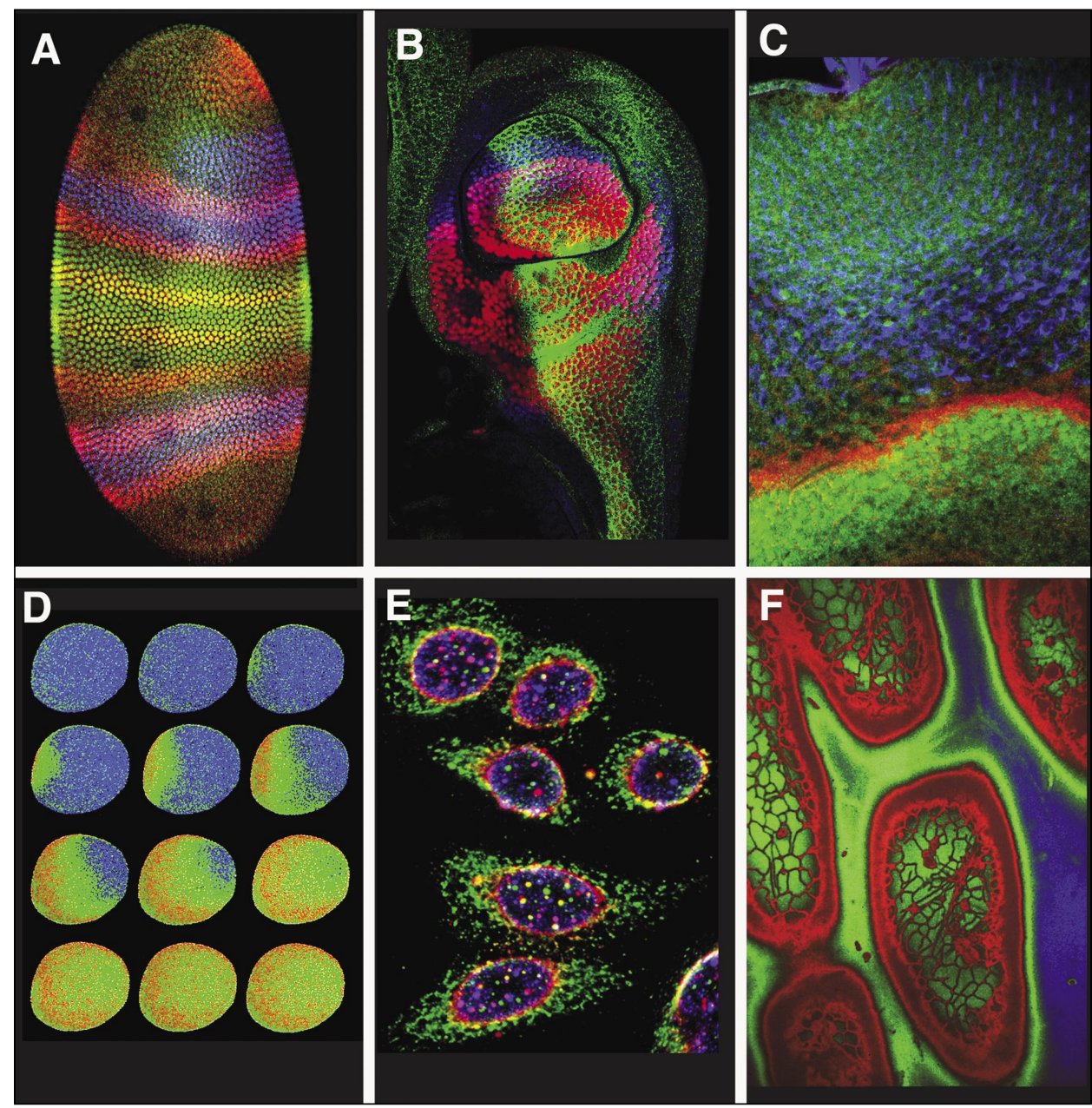

Figure 1. A collection of colorful images that have appeared on the cover of BioTechniques. These images demonstrate some of the applications of confocal microscopy together with the use of color for image display (52). (A) Triple-label Drosophila embryo at the cellular blastoderm stage (53). The seven stripes of hairy expression (in red), together with regions of Kruppel expression (in green), and giant expression (in blue). Here regions of overlap of gene expression are immediately viewed as an additive color change (e.g., the two yellow stripes of hairy in the Kruppel domain). (B) Triple-label Drosophila haltere imaginal disc. (C) Triple-label third instar Drosophila eye imaginal disc. (D) Time-lapse sequence of a calcium wave during starfish fertilization (54). (E) Fluorescently labeled DNA inside the nucleus. Z-series colored for depth. (F) Live cell imaging —endoplasmic reticulum in the water plant Elodea sp. 
laser light to produce an image, and the levels of light that were required to produce acceptable images were phototoxic to many living samples. A second problem was that the signal was attenuated when imaging deeper into the specimen, so that for many specimens, it was impossible to image below two or three cell layers without using damagingly high laser powers.

The phototoxic effects have been gradually reduced over the years by the development of more efficient systems - again by the incorporation of new technology-improved mirrors and more efficient detectors in tandem with the development of brighter and more photostable probes for living samples (e.g., GFP and DsRed) (25). The second problem, that of imaging deeper into specimens, was inherent to the confocal approach. This has been greatly improved with the introduction of multiple-photon imaging $(26,27)$. Using this technique, images can be collected from approximately twice the depth into the specimen as those collected using the confocal approach (28). One of the attractions of the multiple-photon approach is that it uses the same scanning device as the confocal microscope, but a different laser: a rather expensive, yet effective, femtosecond-pulsed laser. Two-photon excitation can excite a fluorophore with a femtosecond time scale. This means that longer wavelength light can be used for the excitation, which for living cells, means less photodamage, and since the two-photon effect only occurs at the plane of focus, less photobleaching of the fluorescent probe. Moreover, longer wavelength excitation is used that can penetrate more deeply into biological tissues. Multiple-photon imaging is now chosen for many live cell applications (Figure 2), and at least one of them has achieved the goals set by the early pioneers of the 1950s, Marvin Minsky and Mohimir Petran, of imaging living neurons in the adult brain (29-31).

There is a depth limit (around $100 \mu \mathrm{m}$, depending on the specimen) at which multiple-photon imaging is not practical. A recent approach taken to image much deeper into specimens, "all-optical histology" has used a femtosecond laser at low power (0.1- to 1.0-nJ pulses) for imaging and then at higher power (1.0- to $10.0-\mu \mathrm{J}$ pulses) to physically remove the imaged layer of tissue and then image the next layer at low laser power (32). In this way, the entire depth of a mouse neocortex was imaged. Deeper imaging at micron resolution has also been achieved using magnetic resonance imaging (MRI), and this approach is becoming more useful with the development of specific probes that are visible using MRI $(33,34)$.

\section{WHICH MICROSCOPE?}

There are now a great variety of choices available for producing optical sections of tissue. Along with confocal microscopes (single-beam scanning LSCMs or multiple-beam scanning Nipkow style) and multiple-photon microscopes, digital deconvolution of wide field images is also a practical option for collecting optical sections (35). More recent methods include a device called the apotome (36) and microMRI $(33,34)$.

There are two major advantages to using confocal microscopy in preference to conventional light microscopy for imaging fluorescently labeled specimens. The first is that the spatial filtering of the pinhole reduces the amount of glare from the specimen. In general, by reducing the diameter of 

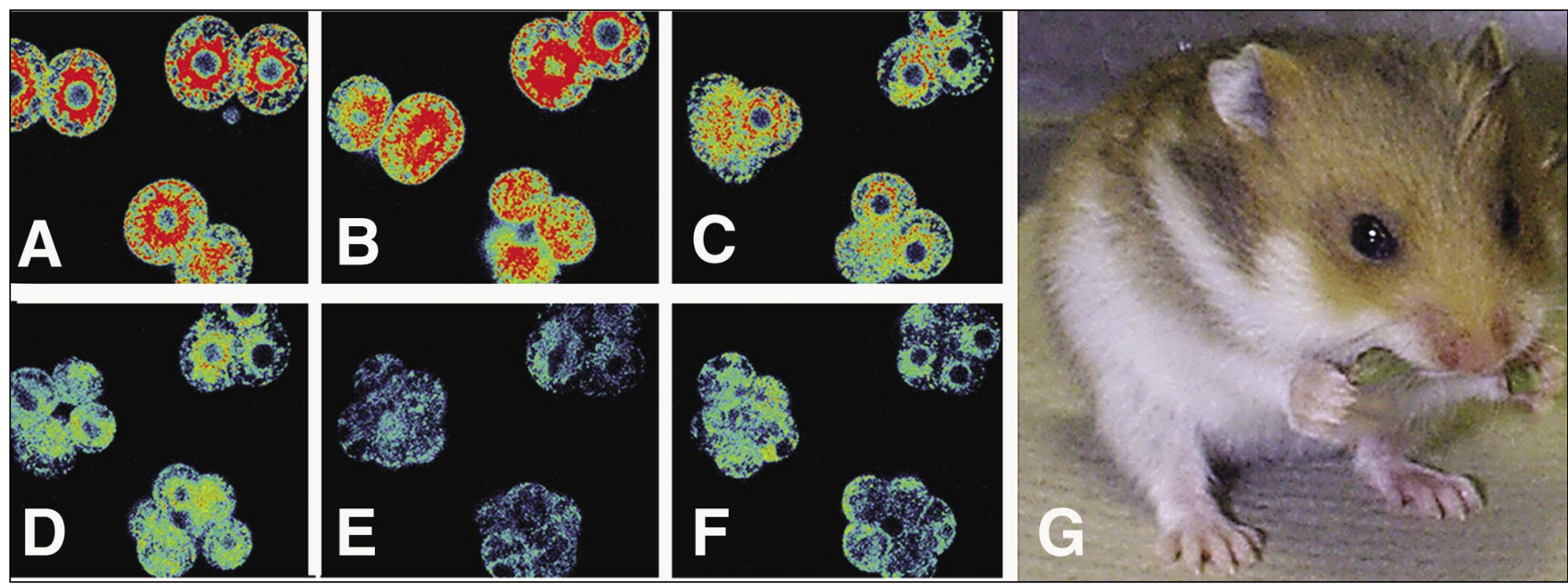

Figure 2. Multiple-photon imaging. Two-photon time-lapse series of three living hamster embryos labeled with the mitochondrial dye MitoTracker ${ }^{\circledR} \mathrm{X}-\mathrm{Rho}-$ damine. The three embryos were imaged every 5 min over a 24-h time period. (A-F) Labeled embryos are shown starting at the 2-cell stage (A) and developing through to the 8-cell stage (F). One of the three embryos was transferred to an adult female who later produced "Laser" shown in (G) when she was 18 days old - a living testament to the improved viability capabilities of multiple-photon imaging. Reproduced with permission from Reference 31.

the pinhole, the amount of specimen sampled is reduced, which gives a thinner optical section. There is an optimal pinhole size for every objective lens. The second major advantage of the confocal microscope is that image resolution is improved by a factor of two if the input and output apertures are both imaged as a diffraction-limited spot.

These factors vary with the objective lens and with the biological specimen. Generally, it is better to use a planapochromat objective lens with the highest possible numerical aperture and no phase rings. Some specimens (e.g., the flattened leading edge of a fibroblast growing in tissue culture and stained with a fluorescent dye) are not improved dramatically by confocal microscopy, whereas thicker specimens with significant 3-D structure (e.g., fluorescently labeled microtubules within multicellular embryos) can only be visualized using confocal microscopy.

Several studies have compared the performance of confocal, multiple-photon, and wide-field deconvolution microscopy on a variety of fluorescently labeled specimens $(24,37,38)$. The current consensus is that wide-field deconvolution is better for thin and relatively dim samples, whereas the LSCM and multiplephoton imaging are preferred for imaging thick and bright samples (e.g., whole mounts of tissues and embryos). The multiplephoton approach is preferred to confocal microscopy for imaging deeper into living tissues. The multiple-beam scanning confocal microscopes are good for imaging faster events at full resolution, whereas the LSCMs can be used for measuring fast events at low resolution. Several studies of living cells have combined the confocal and the deconvolution technique (13). The bottom line is that the choice really depends on the application. However, practical aspects such as instrument availability, ease of use, and cost often determine the final choice of imaging system.

\section{FUTURE CHALLENGES}

The past 20 years of the development of light microscopy have been characterized by talented scientists who have de- veloped instruments for solving specific biological problems (39). Once the microscopes have become more widely available through commercial vendors, the instruments have usually found many additional applications to the intended one. These developments are eventually fed back into later models of the commercially available instruments. Most of the hardware has been imported from elsewhere, and progress has been accelerated by the development of new probes of biological activity, especially those for imaging living cells (40-42).

The challenges of the future are many, and fashions are hard to predict, although most developments are directed towards experiments on living cells in situ (43). Imaging events inside the living nucleus (44-46) remains as one of the greatest challenges some 50 years after Rosalind Franklin's infamous "Photograph 51" was collected (2). Methods of integrating different microscopical techniques including electron microscopy will enhance such studies (47). In fact, electron microscopy is in the process of its own renaissance $(48,49)$ with the development of improved fixation methods (fast-freezing) and image presentation (electron microscope tomography).

Modern microscopes very quickly produce large amounts of data, since hundreds of images can be acquired in a multidimensional experiment. Methods of dealing with such data sets are being developed, including the introduction of universal file formats and international image databases for sharing and comparing data from different laboratories around the world $(50,51)$.

\section{ACKNOWLEDGMENTS}

Thanks to Jim Langeland, Jim Williams, Nadean Brown, Steve Stricker, Jim Hagstrom, Jim Ludtke, and Bill Russin for providing original images (Figure 1) used on BioTechniques' covers and to Sean Carroll and the Howard Hughes Medical Institute (HHMI) for support. Figure 2 was reproduced by permission of Jayne Squirrell and Barry Bavister (copyright holders), University of Wisconsin, Madison. 


\section{REFERENCES}

1.Ellingboe, J. 2003. Twenty years of BioTechniques. BioTechniques 34:452459.

2.Dennis, C. and P. Campbell. 2003. The double helix-fifty years. Nature 421:395-453.

3.Gall, J.G. 1996. Views of the Cell: A Pictorial History. The American Society for Cell Biology, Bethesda.

4.Anderson, K.V. and P.W. Ingham. 2003. The transformation of the model organism: a decade of developmental genetics. Nat. Genet. 33(Suppl):285293.

5.Hurtley, S.M. and L. Helmuth. 2003. Biological imaging. Science 300: 75-102.

6.Schuldt, A. and R. Smallridge. 2003. Imaging in cell biology. Nat. Cell Biol. 6(Suppl):SS1-SS21.

7.Minsky, M. 1988. Memoir on inventing the confocal scanning microscope. Scanning 10:128-138.

8.Minsky, M., inventor. Microscopy apparatus. U.S. patent no. 3013467. 1957.

9.Graves, D.J., H.J. Su, S. Addya, S. Surrey, and P. Fortina. 2002. Fourlaser scanning confocal system for microarray analysis. BioTechniques 32 346-354.

10.Ramdas, L., J. Wang, L. Hu, D. Cogdell, E. Taylor, and W. Zhang. 2001. Comparative evaluation of laser-based microarray scanners. BioTechniques 3:546-550.

11.Schulze, A. and J. Downward. 2001. Navigating gene expression using microarrays-a technology review. Nat. Cell Biol. 3:E190-E195.

12.Petran, M., M. Hadravsky, D. Egger, and R. Galambos. 1968. Tandem scanning reflected light microscope. J. Opt. Soc. Am. 58:661-664.

13.Salmon, W.C., M.C. Adams, and C.M. Waterman-Storer. 2002. Dualwavelength fluorescent speckle microscopy reveals coupling of microtubule and actin movements in migrating cells. J. Cell Biol. 158:31-37.

14.Waterman-Storer, C.M. and G. Danuser. 2002. New directions for fluorescent speckle microscopy. Curr. Biol. 12:R633-R640.

15.White, J.G., W.B. Amos, and M. Fordham. 1987. An evaluation of confocal versus conventional imaging of biological structures by fluorescence light microscopy. J. Cell Biol. 105:41-48.

16.Inoue, S. 1995. Foundations of confocal scanned imaging in light microscopy, p. 1-17. In J.B. Pawley (Ed.), Handbook of Biological Confocal Microscopy. Plenum Press, New York.

17.Selvin, P.R. 2000. The renaissance of fluorescence energy transfer. Nat. Struct. Biol. 7:730-734.

18.Van Roessel, P. and A.H. Brand. 2002. Imaging into the future: visualizing gene expression and protein interactions with fluorescent proteins. Nat. Cell Biol. 4:E15-E20.

19.Zicha, D., I.M. Dobbie, M.R. Holt, J. Monypenny, D.Y.H. Soong, C. Gray, and G.A. Dunn. 2003. Rapid actin transport during cell protrusion. Science 300:142-145.

20.Smallcombe, A. 2001. Multicolor imaging: the important question of co-localization. BioTechniques 30:1240-1247.

21.Paddock, S. 2001. Channel surfing: creating different color combinations from multiple-label images. BioTechniques 30:756-761.

22.Dickinson, M.E., G. Bearman, S. Tille, R. Lansford, and S.E. Fraser. 2001. Multi-spectral imaging and linear unmixing add a whole new dimension to laser scanning fluorescence microscopy. BioTechniques 31:12721278 .

23.Paddock, S. 2001. A brief history of time-lapse. BioTechniques 30:283-289.

24.Andrews, P.D., I.S. Harper, and J.R. Swedlow. 2002. To 5D and beyond: quantitative fluorescence microscopy in the postgenomic era. Traffic 3:2936.

25.Stephens, D.J. and V.J. Allan. 2003. Light microscopy techniques for live cell imaging. Science 300:82-86

26.Denk, W. and K. Svoboda. 1997. Photon upmanship: why multiphoton imaging is more than a gimmick. Neuron 18:351-357.

27.Piston, D.W. 1999. Imaging living cells and tissues by two-photon excitation microscopy. Trends Cell Biol. 9:66-69.

28.Centonze, V. and J.G. White. 1998. Multiphoton excitation provides optical sections from deeper within scattering specimens than confocal imaging. Biophysical J. 75:2015-2024.

29.Lendvai, B., E.A. Stern, B. Chen, and K. Svoboda. 2000. Experiencedependent plasticity of dendritic spines in the developing rat barrel cortex. Nature 404:876-881.
30.Brown, E.B., R.B. Cambell, Y. Tsuzuki, L. Xu, P. Carmeliet, D. Fukumura, and R.K. Jain. 2001. In vivo measurement of gene expression, angiogenesis and physiological function in tumors using multiphoton laser scanning microscopy. Nat. Med. 7:864-868.

31.Squirrell, J.M., D.L. Wokosin, J.G. White, and B.D. Bavister. 1999. Longterm two-photon fluorescence imaging of mammalian embryos without compromising viability. Nat. Biotechnol. 17:763-767.

32.Tsai, P.S., B. Friedman, A.I. Ifarraguerri, B. Thompson, V. Lev-Ram, C.B. Schaffer, Q. Xiong, R. Tsien, et al. 2003. All-optical histology using ultrashort laser pulses. Neuron 39:27-41.

33.Lichtman, J.W. and S.E. Fraser. 2001. The neuronal naturalist: watching neurons in their native habitat. Nat. Neurosci. 4(Suppl):1215-1220.

34.Jacobs, R.E., E.T. Ahrens, T.J. Meade, and S.E. Fraser. 1999. Looking deeper into vertebrate development. Trends Cell Biol. 9:73-76.

35.Wallace, W., L.H. Schaefer, and J.R. Swedlow. 2001. Workingperson' guide to deconvolution in light microscopy. BioTechniques. 31:1076-1097.

36.Butkus, B.D. 2003. Microscope accessory creates optical sectioning via structured illumination. BioPhotonics Int. August:19-20.

37.Swedlow, J.R., K. Hu, P.D. Andrews, D.S. Roos, and J.M. Murray. 2002 Measuring tubulin content in Toxoplasma gondii: a comparison of laser-scanning confocal and wide-field fluorescence microscopy. Proc. Natl. Acad. Sci. USA 99:2014-2019.

38.Periasamy, A., P. Skoglund, C. Noakes, and R. Keller. 1999. An evaluation of two-photon excitation versus confocal and digital deconvolution fluorescence microscopy imaging in Xenopus morphogenesis. Microsc. Res. Tech. 47:172-181.

39.Amos, B. 2000. Lessons from the history of light microscopy. Nat. Cell Biol 2:E151-E152

40.Larson, D.R., W.R. Zipfel, R.M. Williams, S.W. Clark, M.P. Bruchez, F.W. Wise, and W.W. Webb. 2003 Water-soluble quantum dots for multiphoton fluorescence imaging in vivo. Science 300:1434-1436.

41.Lippincott-Schwartz, J. and G.H. Patterson. 2003. Development and use of fluorescent protein markers in living cells. Science 300:87-91.

42.Zhang, J., R.E. Campbell, A.Y. Ting, and R.Y. Tsien. 2002. Creating new fluorescent probes for cell biology. Nat. Rev. Mol. Cell Biol. 3:906-918.

43.Tsien, R. 2003. Imagining imaging's future. Nat. Rev. Mol. Cell Biol. 4(Suppl):SS16-SS21.

44.Janicki, S.M. and D.L. Spector. 2003. Nuclear choreography: interpretations from living cells. Curr. Opin. Cell Biol. 15:149-157.

45.Pederson, T. 2002. Dynamics and genome-centricity of interchromatin domains in the nucleus. Nat. Cell Biol. 4:E287-E291.

46.Gasser, S. 2002. Visualizing chromatin dynamics in interphase nuclei. Science 296:1412-1416.

47.Gaietta, G., T.J. Deerinck, S.R. Adams, J. Bouwer, O. Tour, D.W. Laird, G.E. Sosinsky, R.Y. Tsien, and M.H. Ellisman. 2002. Multicolor and electron microscopic imaging of connexin trafficking. Science 296:503-507.

48.McIntosh, J.R. 2001. Electron microscopy of cells: a new beginning for a new century. J. Cell Biol. 153:F25-F32.

49.Medalia, O., I. Weber, A.S. Frangakis, D. Nicastro, G. Gerisch, and W. Baumeister. 2002. Macromolecular architecture in eukaryotic cells visualised by cryoelectron tomography. Science 298:1209-1213.

50.Swedlow, J.R., I. Goldberg, E. Brauner, and P.K. Sorger. 2003. Informatics and quantitative analysis in biological imaging. Science 300:100-102.

51.Marx, V. 2002. Beautiful bioimages for the eyes of many beholders. Science 297:39-40.

52.Paddock, S.W., E.J. Hazen, and P.J. DeVries. 1997. Methods and applications of three-color confocal imaging. BioTechniques 22:120-126.

53.Paddock, S.W., J. Langeland, P. DeVries, and S.B. Carroll. 1993. Three color immunofluorescence imaging of Drosophila embryos by laser scanning confocal microscopy. BioTechniques 14:42-48.

54.Stricker, S.A. 2000. Confocal microscopy of intracellular calcium dynamics during fertilization. BioTechniques 29:492-498.

Address correspondence to:

Stephen W. Paddock

University of Wisconsin

Department of Molecular Biology

The Bock Building

Madison, WI 53706, USA

email: paddock@wisc.edu 